\title{
Investigating effect of water of hydration on active pharmaceutical ingredients in a water-sensitive dosage form
}

Monica C. Chuong*, Charles J. Kelley, Yosra Muhammad, Thais Dias Caputo, Jader M. Gomes, Denise Oliveira, Andiara Cardoso Peixoto, Bruna Sanches Pereira, Waleed Rizg, Carolina Vazquez, Thiago Medeiros Zacaron, Steven Nguyen and David A. Williams

\begin{abstract}
Background: Preparation of mini buccal tablets incorporating either of the Alzheimer's drugs, rivastigmine tartrate or donepezil hydrochloride, were developed for patients who have difficulty swallowing as a source of effervescence, by pairing the less commonly used malic acid with sodium bicarbonate in an experimentally determined (1:2) stochiometirc ratio.

Methods: To avoid premature reaction of the acid and the base during compounding, the tablet ingredients were mixed in the following order: acid, sweetener, binder, drug, preservative, base, and anti-adherent/lubricant.

Results: An accelerated thermal stability study at $40{ }^{\circ} \mathrm{C}$ and $25 \%$ relative humidity showed that the integrity of the effervescent tablets containing rivastigmine tartrate were superior to that of donepezil $\mathrm{HCl}$ tablets. FT-IR spectrometry confirmed the presence of water of hydrate in donepezil $\mathrm{HCl}$ crystals. This water was absent in the IR after one-month storage at accelerated thermal stability, but was present at room temperature. This behavior was not observed in the tablet made from rivastigmine tartrate powder. Differential scanning calorimetry of donepezil hydrochloride showed two thermal events: the first was associated with the loss of the water, and the second, at much higher temperature, was the melting of theanhydrous drug. Such behavior was not observed in the rivastigmine tartrate powder.
\end{abstract}

Conclusions: Hidden water which may function as catalyst to induce premature effervescence during storage.

Keywords: Friability, Hardness, Differential scanning calorimetry, Donepezil $\mathrm{HCl}$, Fourier-transform infrared spectroscopy, Malic acid, Rivastigmine tartrate

\section{Background}

Effervescent tablets or granules contain mixtures of acid(s) (e.g., citric acid, tartaric acid) and base(s) (sodium or potassium bicarbonate/carbonate). Upon contact with water, these formulations have the capability to react with each other and liberate carbon dioxide gas resulting in the effervescent action (Pharmaceutical Dosage Forms, General Chapters, 2017; Bertuzzi, 2009; Shrewsbury, 2015). For some active ingredients that are not stable for compounding into liquid form, oral solution or suspension may be administered to the patients who have difficulties in swallowing by prior dissolution of the

\footnotetext{
* Correspondence: monica.chuong@mcphs.edu

Department of Pharmaceutical Sciences, MCPHS University, Boston, MA, 179 Longwood Ave, Boston, MA 02115, USA
}

effervescent tablet or granules in a cup of water. This study investigated both rivastigmine tartrate and donepezil hydrochloride $(\mathrm{HCl})$. Both model drugs are cholinesterase inhibitors for the treatment of dementia in patients with Alzheimer's disease. The approved dosage forms by the US Food and Drug Administration (US FDA) for rivastigmine tartrate are capsules and extended release transdermal film loaded with free drug (http://www.accessdata.fda.gov/scripts/cder/ob/docs/tempai.cfm, 2017). However, rivastigmine tartrate liquid has been discontinued. The approved dosage forms for donepezil $\mathrm{HCl}$ are available as tablets and orally disintegrating tablet (http:// www.accessdata.fda.gov/scripts/cder/ob/docs/tempai.cfm, 2017). This study aimed to investigate the substitution of commonly used effervescent acids (such as citric acid 
and/or tartaric acid) (Bertuzzi, 2009) with a less studied fruit acid (malic acid). This study suggests an improvement strategy.

\section{Methods}

Materials

Rivastigmine L-tartrate ( $\geq 98 \%$ (http://www.tcichemicals.com/eshop/en/us/commodity/D4099/, 2017), TCI, JQEVO-TQ), donepezil HCl ( $\geq 98 \%$ (http://www.tcichemicals.com/eshop/en/us/commodity/R0093/, 2017), TCI, Lot UVA20-PB), acetonitrile, hydrochloric acid (36.5-38\%), malic acid, monobasic ammonium phosphate, methanol, perchloric acid (60-62\%), sodium benzoate, sodium bicarbonate, and tartaric acid anhydrous were purchased from VWR International (Bridgeport, NJ). Polyethylene glycol (PEG) 8000 was obtained from Professional Compounding Company of America (PCCA, Houston, TX). Splenda sweetener was purchased locally. USP-NF 2017 describes donepezil $\mathrm{HCl}$ as anhydrous powder (containing NMT $0.4 \%$ of water), and monohydrate form (>98\%, NMT 7.0\% water). Rivastigmine tartrate contains not more than (NMT) $0.5 \%$ water and total impurity NMT 0.5\% (Pharmaceutical Dosage Forms, General Chapters, 2017).

\section{Methods}

\section{Balance of effervescent reaction}

Citric acid and tartaric acid $(1: 2 w / w)$ coupled with sodium bicarbonate are commonly recommended in effervescence (Bertuzzi, 2009; Shrewsbury, 2015; Allen Jr. \& Howard, 2013) to overcome its tackiness (http://www.accessdata.fda.gov/scripts/cder/ob/docs/tempai.cfm, 2017; Allen Jr. \& Howard, 2013) since the former is rather sticky and the latter produces a chalky friable granule (Allen Jr. \& Howard, 2013) This project investigated the use of malic acid as the effervescent acid instead of the mixture of citric acid and tartaric acid. Malic acid is a naturally occurring acid found in many fruits and vegetables, and is largely responsible for the sour taste found in apples and pears. The chemical reaction was balanced based on stoichiometric coefficients and molecular weights to determine the ratio between malic acid and sodium bicarbonate. The required amounts of malic acid (in grams) and sodium bicarbonate (in grams) were physically mixed according to the predicted ratio, and were allowed to react in water without stirring to observe whether the reaction was complete, and if any residue crystals left to ensure the stoichiometric coefficients had been correctly balanced.

\section{Two initial innovative tablet designs for rivastigmine} tartrate oral solution: effervescence vs. fast disintegration In addition to malic acid and sodium bicarbonate, the excipients used to form a single $9.6 \mathrm{mg}$ rivastigmine tartrate effervescent tablet (equivalent to $6 \mathrm{mg}$ drug base) were sweetener (Splenda), preservative, and polyethylene glycol (PEG) 800. Each excipient was individually triturated into fine powder to avoid acid-base reaction upon contact. Ingredients were added in different order to keep acid and base apart, and gently mixed. Rivastigmine tartrate fast disintegration tablets for oral solution was also formulated similarly.

The mixture was dry compressed by a single punch machine (GlobePharma, MTC4-1) in two different punch surface designs by Notoli (Notoli Descriptions of Goods, 2017) at different pressures to identify an optimal hardness and comply with friability test. Excipients modification continued until a tablet candidate passed friability test (ERWEKA Gmbh, Piscataway Township, NJ, model TDR 100) for rolling and impact durability. According to General Chapters: <1216> Tablet Friability in USP-NF, friability is defined as percentage weight loss from a tablet, which should not be more than $1 \%$ (Pharmaceutical Dosage Forms, General Chapters, 2017). The tablets were then subjected to a hardness tester (Schleuniger, New York, NY, model 2E). The large size of a traditional effervescent tablet $(>1.5 \mathrm{~cm})$ allows visual examination of the tablet breakage pattern without having to use a microscope (Notoli Descriptions of Goods, 2017; Aulton \& Taylor, 2013) to eliminate the disturbance of the breakage pattern.

\section{Formulation of mini effervescent tablets to disintegrate in oral cavity using Rivastigmine Tartrate and Donepezil $\mathrm{HCl}$ respectively as module drug}

Formualtion, hardness, and friability tests This project altered fabrication from traditional effervescent tablet to effervescent mini buccal tablet size to reduce the sodium content in the required sodium bicarbonate to make into such a tablet. For effervescent mini buccal tablets, the tablet total weight was designed at approximately $100 \mathrm{mg}$ which contained much less amount of sodium content. All components and compositions of effervescent mini buccal tablets were kept the same, except the model drugs were loaded as $9.6 \mathrm{mg}$ rivastigmine tartrate (equivalent to $6 \mathrm{mg}$ drug base) and $10.7 \mathrm{mg}$ donepezil $\mathrm{HCl}$ (equivalent to $10 \mathrm{mg}$ drug base) according to FDA orange book (http://www.accessdata.fda.gov/ scripts/cder/ob/docs/tempai.cfm, 2017). Tablet friability and the hardness tests were performed as described in Section 2.2.2 for testing traditional effervescent tablets.

Performance tests There are no disintegrating performance tests set in USP-NF for mini effervescent tablet. Therefore, we tested the disintegration and dissolution of different formulas in test tubes, then quantified the drug content using HPLC. The HPLC assays for donepezil $\mathrm{HCl}$ 
as well as rivastigmine tartrate mini buccal tablets were taken USP monographs (Pharmaceutical Dosage Forms, General Chapters, 2017). The mobile phases were prepared accordingly.

Accelerated product stability study The 30 -day accelerated stability study was performed to obtain useful information for screening workable candidates. Effervescent mini buccal tablets of donepezil $\mathrm{HCl}$ and rivastigmine tartrate created in the lab were randomly divided into two groups respectively. Each group was placed in a 4oz amber glass bottle and sealed tightly with parafilm. One group from each formulation batch was stored in an incubator (Fisher Scientific, Waltham, MA, model 11-690-625D) at 25\% relative humidity (RH) and $40 \pm$ $2{ }^{\circ} \mathrm{C}$, while the second group was stored at controlled RT $\left(25 \pm 2{ }^{\circ} \mathrm{C}\right)$, and $60 \% \mathrm{RH}$ according the worldwide climatic zone II: Subtropical and Mediterranean climate defined by WHO to which the USA belongs to (Notoli Descriptions of Goods, 2017). The relative humidity and temperature were monitored by two laboratory hygrometers.

\section{Fourier transform infrared spectrometry and differential scanning calorimetry}

Fourier transform infrared spectrometry (FT-IR) and differential scanning calorimetry (DSC) technology were used to verify purity and to assist in explaining any instability. The protocols of both are briefly described below.

FT infrared spectrometry A FT-IR spectrometer with OMNIC $^{\text {ma }}$ Series Software (Thermo Scientific) was used to examine and compare the powder obtained from a crushed effervescent mini buccal tablet with its pure donepezil and rivastigmine drug, excipient powder, and each excipient used in formulation in the wave number region between 4000 and $400 \mathrm{~cm}^{-1}$ (Silverstein et al, 2015).

Differential scanning calorimetry Prior to DSC study, the melting temperature of each excipient used in effervescent mini buccal tablet formulations was obtained from literature. A DSC (SSC-5200-Seiko Instruments, Cheshire, UK) linked to the EXSTAR Station and a cooling controller equipped with liquid nitrogen was used to conduct thermal analyses of donepezil $\mathrm{HCl}$ and rivastigmine tartrate drug powders, excipient powders, and the powder from each crushed effervescent mini buccal tablet. The powder was weighed using a DSC standard aluminum sample pan and lid in a weight range of 3$5 \mathrm{mg}$. The same pan and lid pair assembled without powder within was placed on the reference stove. The incremental temperature was set at $10{ }^{\circ} \mathrm{C} / \mathrm{min}$ from RT to a predetermined temperature. For example, the scans of donepezil $\mathrm{HCl}$ ended at $250{ }^{\circ} \mathrm{C}$, while rivastigmine tartrate ended $125{ }^{\circ} \mathrm{C}$ according to their melting temperatures listed in the literature (Pharmaceutical Dosage Forms, General Chapters, 2017).

\section{Results}

Formulation of conventional tablet for oral solution and balance of effervescent reaction

Two types of rivastigmine tartrate tablets for oral solution formulation were initially prepared: effervescence and fast disintegration. The ingredients incorporated are listed in Table 1. The effervescent tablet was fabricated with malic acid and sodium bicarbonate. The stoichiometric coefficients of the chemical reaction were balanced as Eq. 1.

$$
\begin{aligned}
& \mathrm{C}_{4} \mathrm{H}_{6} \mathrm{O}_{5} \text { (malic acid) }+2 \mathrm{NaHCO}_{3} \text { (sodium bicarbonate) } \\
& \rightarrow 2 \mathrm{H}_{2} \mathrm{O}+2 \mathrm{CO}_{2}+\mathrm{Na}_{2} \mathrm{C}_{4} \mathrm{H}_{4} \mathrm{O}_{5}
\end{aligned}
$$

Malic acid (in grams) and sodium bicarbonate (in grams) were physically mixed according to the predicted ratio in Eq. 1 and were then allowed to react in water to completion without stirring to ensure that the stoichiometric coefficients in Eq. 1 are correct. The ingredients for formulating the tablets (Table 1) were added in the following order: acid, sweetener, binder, drug, preservative, base, and tableting antiadherent/lubricant; and gently mixed with a spectula in a mortar. For the fast disintegration tablet, because no chemical reaction was expected to trigger the effervescence, the principle of geometric dilution was used to mix all ingredients together (Table 1) after each was triturated to fine powder.

\section{Hardness and friability tests}

Tablets were compressed by flat surface punch set without bevel edge failed the friability test. Contradictorily, those compressed by punch set with bevel edge required less binder and formed thinner tablets $(5.7 \mathrm{~mm}, 1.73 \mathrm{~g}$ ) and passed the friability test. The effervescent tablets that were compressed with the punches without bevel edge

Table 1 The excipients and pharmaceutical functions of effervescent tablet (F1) and fast disintegrating tablet (F2)

\begin{tabular}{lccl}
\hline Excipient & F1 & F2 & Pharmaceutical function \\
\hline Malic acid & $\checkmark$ & & Acid \\
Citric acid & & $\checkmark$ & Acid \\
Sodium bicarbonate & $\checkmark$ & & Base \\
Sodium citrate & & $\checkmark$ & Acid salt \\
Sucralose & $\checkmark$ & $\checkmark$ & Sweetener \\
Cellulose microcrystalline & & $\checkmark$ & Binder \\
Sodium chloride & & $\checkmark$ & Disintegration enhancer \\
PEG & $\checkmark$ & $\checkmark$ & Binder \\
Sodium Benzoate & $\checkmark$ & $\checkmark$ & Preservative \\
\hline
\end{tabular}


(Notoli Descriptions of Goods, 2017) lost more than 1\% weight thus failed the friability test (Pharmaceutical Dosage Forms, General Chapters, 2017). The breakage was mainly due to simple tensile failure with slightly shear failure at one platen edge (Aulton \& Taylor, 2013; Bubb, 2011).

\section{Formulation of effervescent mini buccal tablets}

The effervescent mini buccal tablets were designed at approximate $100 \mathrm{mg}$ total weight containing either $9.6 \mathrm{mg}$ of rivastigmine tartrate (equivalent to $6 \mathrm{mg}$ drug base) or $10.7 \mathrm{mg}$ of donepezil $\mathrm{HCl}$ (equivalent to $10 \mathrm{mg}$ drug base), while all excipients were kept the same except the lubricant was chosen from among magnesium stearate, stearic acid, and tartaric acid. The components, compositions, and pharmaceutical function of each formulation are listed in Table 2.

\section{Hardness, friability and performance tests for Ddonepezil $\mathrm{HCl}$ effervescent mini buccal tablets}

The compression loss, tablet diameter and thickness, friability loss and hardness tests of the effervescent buccal donepezil $\mathrm{HCl}$ tablet (2a) were $1.26 \pm 0.47 \%, 0.25 \pm$ $0.02 \mathrm{~cm}, 0.122 \pm 0.002 \mathrm{~cm}$ (center thickness), $0.078 \pm$ $0.009 \mathrm{~cm}$ (edge thickness), $0.38 \pm 0.20 \%$, and $4.25 \pm 0.85$ $\mathrm{kp}$, respectively $(n=6)$. When water was used as the disintegrating medium as suggested in USP-NF (Pharmaceutical Dosage Forms, General Chapters, 2017), the tablet disintegrating time in a test tube containing $5 \mathrm{~mL}$ of water was $1 \mathrm{~min} 51 \mathrm{~s}(n=6)$. The chromatographic retention time of donepezil $\mathrm{HCl}$ was $12.4 \mathrm{~min}$ (data not shown).

\section{Hardness and friability tests for rivastigmine tartrate effervescent mini buccal tablets}

The compression loss, friability loss and hardness tests of the effervescent mini buccal rivastigmine tartrate tablets formed at 1500 psi compression pressure were
$1.013 \pm 0.633 \% ; 0.100 \pm 0.082 \% ; 2.23 \pm 0.38$ kilopond $(\mathrm{kp})$ respectively $(n=4)$. Among the three antiadherents/lubricants (magnesium stearate, stearic acid, and tartaric acid) incorporated for tableting, their tablet disintegrating dispersions passed through a No. 25 sieve (aperture $0.71 \mathrm{~mm}$ ) as a part of performance test for effervescent dosage form set by USP compendium (Pharmaceutical Dosage Forms, General Chapters, 2017). The solution of the batch containing $3.17 \%$ tartaric acid was totally clear, but not the liquid containing disintegrated tablets made of magnesium stearate or stearic acid. The retention time of rivastigmine HPLC was $6.8 \mathrm{~min}$ (data not shown). Judging from the breakage pattern of our successful rivastigmine tartrate mini buccal tablets, failure caused by diametrical compression was also due to the tensile failure.

\section{Stability accelerated tests}

The appearances of both donepezil $\mathrm{HCl}$ and rivastigmine tartrate effervescent buccal tablets after 1 month of stability study at $40{ }^{\circ} \mathrm{C}$ at $25 \% \mathrm{RH}$, and controlled RT $\left(23 \pm 2{ }^{\circ} \mathrm{C}\right)$ at $60 \% \mathrm{RH}$ are shown in Fig. 3. Both tablet exhibited discoloration (Fig. 1a, c). The tablet integrity of donepezil $\mathrm{HCl}$ (Fig. 1a) at $40{ }^{\circ} \mathrm{C} 25 \% \mathrm{RH}$ exhibited efferescence, whereas the tablets at room temp remained unchanged (Fig. 1b). On the other hand, rivastigmine tartrate tablets (Fig. 1c, d) exhibited no significant visual change in shape, except color. FT-IR and DSC of donepezil tablet from the $25 \% \mathrm{RH}$ and $40{ }^{\circ} \mathrm{C}$ were further applied to explore the cause.

\section{FT- infrared and DSC scans}

As aforementioned (Section 3.2 and Table 2), all excipient components and composition of effervescent mini buccal tablets of donepezil $\mathrm{HCl}$ and rivastigmine tartrate were the same, except the drug content (10.7 and

Table 2 Components, compositions, and pharmaceutical functions of rivastigmine tartrate and donepezil $\mathrm{HCl}$ effervescent mini buccal tablets

\begin{tabular}{|c|c|c|c|c|}
\hline \multirow[t]{2}{*}{ Ingredient } & Donepezil & Rivastigmine & Rivastigmine & Pharmaceutical \\
\hline & Buccal tablet & Buccal tablet (F1) & Buccal tablet (F2) & Function \\
\hline Rivastigmine tartrate & - & 9.6 & 9.6 & API \\
\hline Donepezil HCl & 10.7 & - & - & API \\
\hline Malic acid & 20.71 & 20.71 & 20.71 & Acid \\
\hline Sodium bicarbonate & 25.95 & 25.95 & 25.95 & Base \\
\hline Splenda & 33.5 & 33.5 & 33.5 & Sweetening \\
\hline Sodium benzoate & 0.24 & 0.24 & 0.24 & Preservative \\
\hline PEG 8000 & 10.65 & 10.65 & 10.65 & Binder \\
\hline Magnesium stearate & - & 2.37 & - & Lubricant \\
\hline Tartaric acid & 3.4 & - & 3.4 & Lubricant \\
\hline Total Weight & 107.2 & 103.9 & 106.53 & \\
\hline
\end{tabular}




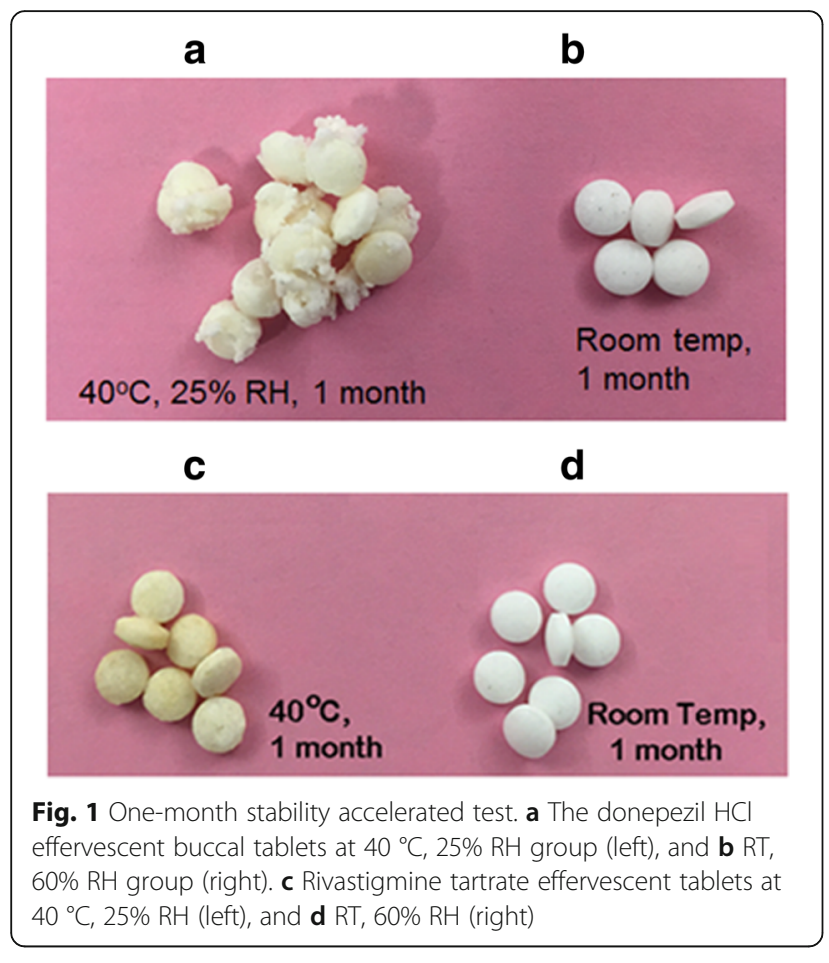

$9.6 \mathrm{mg}$, respectively). The freshly compressed tablets of both groups were crushed into powder for FT-IR and DSC studies.

FT infrared spectrometry The FT-IR scans of both drug crystals, excipients (malic acid, tartaric acid, sodium bicarbonate, Splenda, PEG 8000 and sodium benzoate), and the crushed powder from formulated tablet groups were scanned. The FT-IR of pure donepezil $\mathrm{HCl}$ revealed a sharp peak at $3586 \mathrm{~cm}^{-1}$ (Fig. 2a), which corresponded to water of hydration. The IR peak for the water of hydration had disappeared after the tablets were aged at $40{ }^{\circ} \mathrm{C}$ and $25 \%$ $\mathrm{RH}$ (the fourth trace of Fig. 2a). The IR scans were dominated by the excipients and were also present in the spectrum of pure Splenda (Fig. 2b). The IR spectra of pure rivastigmine (the first trace of Fig. 3) and the crushed powder of its tablet (second to fourth the traces of Fig. 3) peaks observed in the tablet's spectrum were also dominated by Splenda.

Differential scanning calorimetry The melting temperatures of the excipient used in effervescent mini buccal tablet formulation were: malic acid is $131-132{ }^{\circ} \mathrm{C}$, sodium bicarbonate $270{ }^{\circ} \mathrm{C}$, Splenda (sucralose $130{ }^{\circ} \mathrm{C}$, dextrose $146{ }^{\circ} \mathrm{C}$, maltodextrin $240{ }^{\circ} \mathrm{C}$ ), PEG 8000 60-63 ${ }^{\circ} \mathrm{C}$, sodium benzoate $410{ }^{\circ} \mathrm{C}$ and tartaric acid 168-170 ${ }^{\circ} \mathrm{C}$ (Shrewsbury, 2015; Rowe et al., 2009).

(a) Donepezil $\mathrm{HCl}$
The donepezil $\mathrm{HCl}$ scan (the red trace of Fig. 4a) showed that the compound continued to absorb heat until $116.2{ }^{\circ} \mathrm{C}$, and then changed to a stable polymorph until reaching its phase transition point at $225.2{ }^{\circ} \mathrm{C}$, its melting point. Combined with the information of FT-IR, this behavior was due to the loss of the water of hydration which had been trapped in the donepezil $\mathrm{HCl}$ crystal during manufacturing stage. As to the donepezil $\mathrm{HCl}$ crushed tablet scan (the blue and green traces of Fig. 4a), the scans exhibit the behavior of the excipients, most likely the PEG, and the Splenda substances. The phase transition of PEG $8000 \mathrm{mp}$ is at $60-63{ }^{\circ} \mathrm{C}$. The depression at $120-180{ }^{\circ} \mathrm{C}$ were probably related to malic acid, sucralose, dextrose, and tartaric acid. The brown discoloration can be attributed to interaction with the alkalinity of the bicarbonates with the carbohydrates in Splenda.

\section{(b) Rivastigmine Tartrate}

The DSC traces of drug powder overlapped with tablet crushed powder illustrated that the rivastigmine tartrate powder used in the formulation was a pure compound (the red trace in Fig. 4b). The melting range of rivastigmine tartrate in its tablet scan (the blue and green and blues traces of Fig. 4b) was broader than that in the pure drug scan (the red trace of Fig. 4b). The melting temperature of each excipient, which was incorporated in forming effervescent mini buccal tablets, was further searched from literature to seek for the explanation of the DSC endothermic event at $59.8^{\circ} \mathrm{C}$ in the tablet scan (the blue and green traces in Fig. 4b), but not seen in the drug compound (red trace). It was probably due to the phase transition of PEG 8000. The second endothermic event of rivastigmine tablets which was rather board between 120 to $140{ }^{\circ} \mathrm{C}$ were probably due to melting of malic acid and sucralose $\left(\mathrm{mp} 130{ }^{\circ} \mathrm{C}\right)$, dextrose $\left(\mathrm{mp} 146^{\circ} \mathrm{C}\right)$ in Splenda.

\section{Discussion}

\section{Process concerns}

A traditional effervescent tablet has to be unswallowable in diameter to avoid the undesirable side effects from aggressive gas generation such as gastric distension or small intestinal perforation. The balance between the binder and disintegrant requirements for these big tablets is challenging. Binder ensures passing friability rolling and impact durability, but delays the completion of disintegration within $3 \mathrm{~min}$.

Very little water should be used as an ingredient, or be encountered during compounding and handling effervescent granules and tablets. It is advised to process effervescent tablets in an environment of NMT 25\% RH and controlled room temperature (Bertuzzi, 2009). 


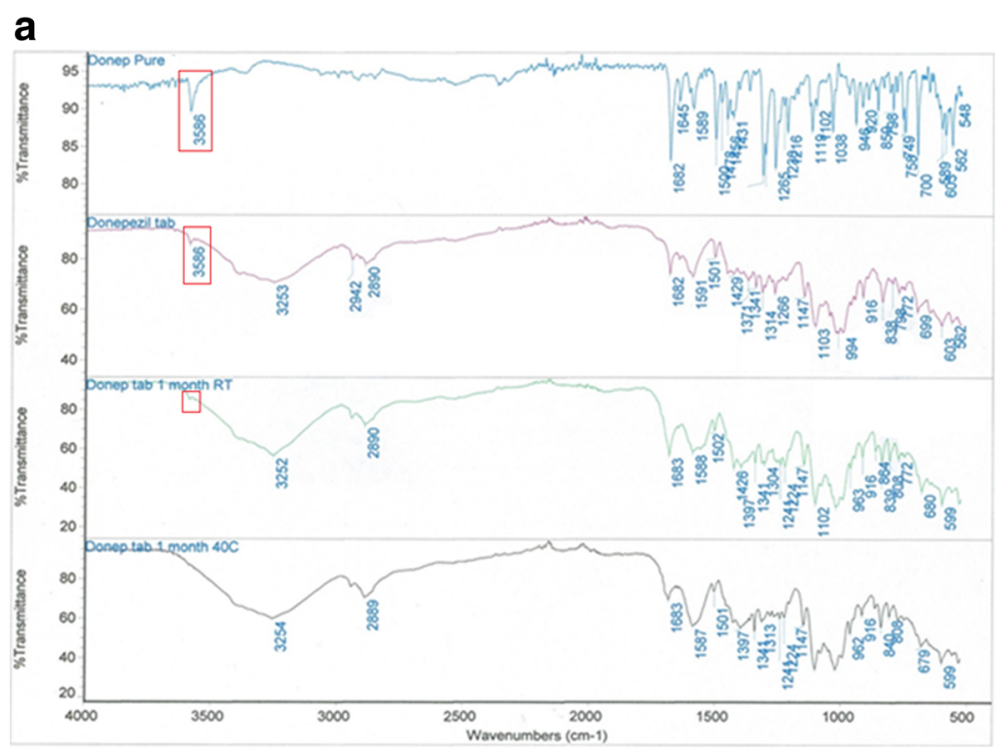

b

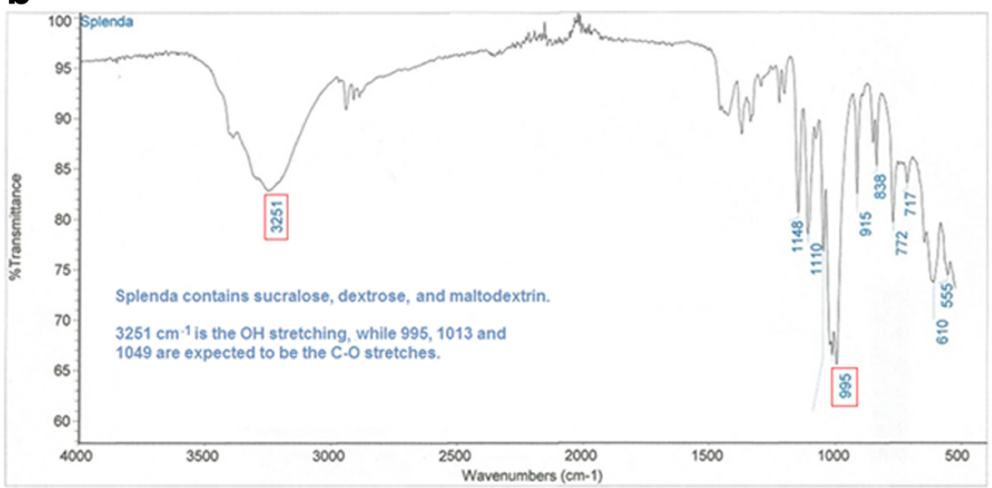

Fig. 2 The FT-IR spectra of donepezil $\mathrm{HCl}$. a The donepezil $\mathrm{HCl}$ crystals and the crushed tablet after storage at controlled $\mathrm{RT}, 60 \% \mathrm{RH}$ vs. $40{ }^{\circ} \mathrm{C}$, and $25 \%$ RH for 1 month. Apparently, the water of hydration had reacted with the acid and base excipients giving off carbon dioxide. $\mathbf{b}$ Splenda

Granules should be packaged in individual pouches, while tablets be packaging with desiccants in thin tall tubes with internal polymeric coat and hermetic seal to insure complete protection. Quantities sufficient for a limited time period use, such as for 2 weeks, are also advised (Pharmaceutical Dosage Forms, General Chapters, 2017). The label of "Not to open until the time of use" should also be included. Otherwise, they may self-destruct due to the effervescent reaction catalyzed by the water vapor present inside a container. When pharmaceutical scientists purchase raw materials, caution for the impurity being trapped in during manufacturing and purification processes must be taken. The impurity components and compositions should be made available in the safety data sheet of a raw material. Detection of hydrate present in a compound may be done with DSC combined with thermogravimetric analysis (TGA), HPLC or FTIR in comparison with its USP Reference Standard.

\section{Accelerative study concerns}

Even though rivastigmine tartrate crystal contained no water, as shown in the accelerated stability study at $40{ }^{\circ} \mathrm{C}$ (the forth trace in Fig. 3), carbonyl browning was still present in the tablet (Fig. 1c). Therefore, the accelerated stability study to determine the shelf life is suitable for this dosage form for ingredient screening. When determining the shelf life for this dosage form, it is recommended to perform this study at controlled RT and relative humidity according the worldwide climatic zone defined by WHO (Aulton \& Taylor, 2013), where the products will be marketed.

\section{Malic Acid versus Maleic Acid}

Although malic acid and maleic acid are similar in spelling, they differ in their chemical structures and physiochemical properties (http://www.newsmax.com/FastFeatures/malic- 


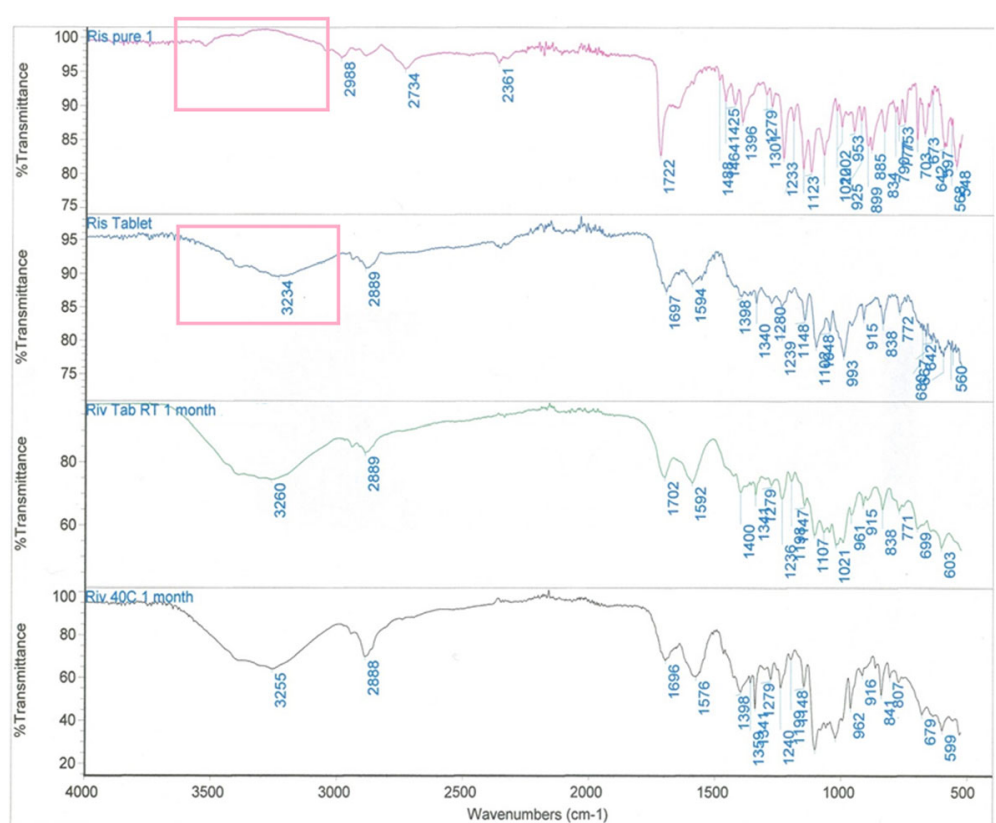

Fig. 3 The FT-IR spectra of rivastigmine tartrate crystals and the crushed tablet after storage at controlled RT, $60 \%$ RH vs. $40{ }^{\circ} \mathrm{C}$, and $25 \%$ RH for 1 month. No sign of water of hydration had reacted with the acid and base excipients giving off carbon dioxide

acid-benefits-foods-highest-concentration-diet/2015/04/01/ id/635741/, 2017; https://en.wikipedia.org/wiki/Maleic_acid, 2017; New Jersey Department of Health and Senior Services Hazardous Substance Fact Sheet, 2017; Pharmaceutical Dosage Forms, General Chapters, 2017).

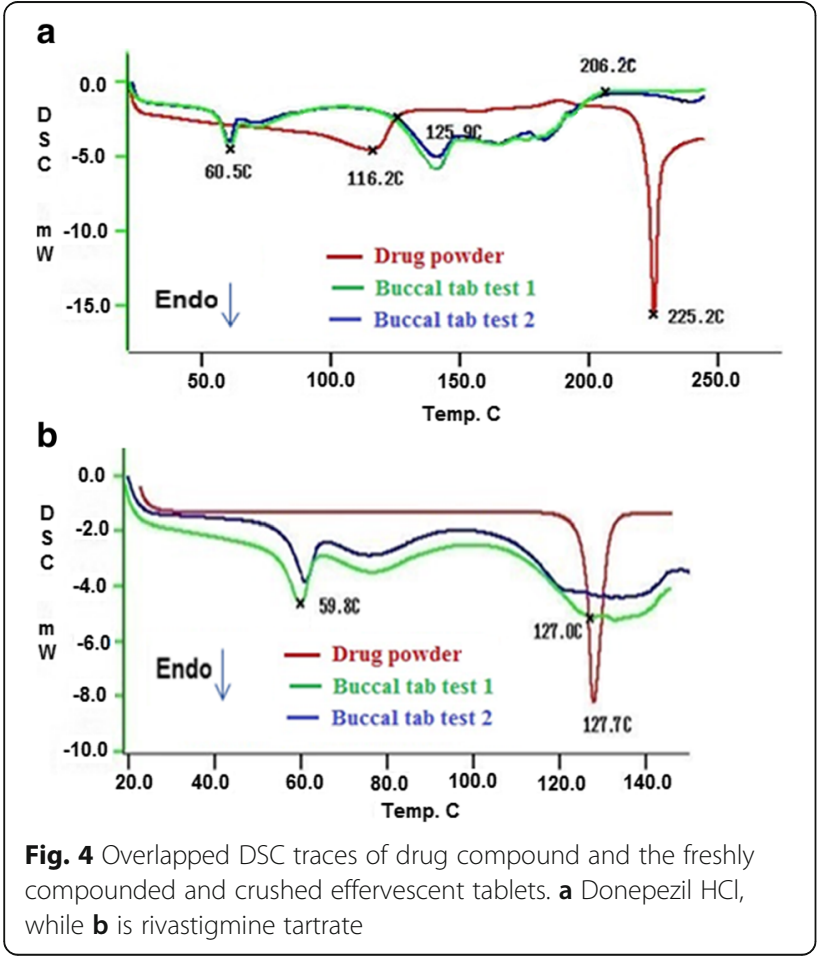

Malic acid may be used as an effervescent acid, but not maleic acid. The toxic renal effect of maleic acid had been reported (Vega \& Colinas, 2009).

\section{Absorption pathways for effervescent buccal tablets}

OraVescent Drug Delivery system: A Novel Technology for Transmucosal Delivery of Drugs Elucidated OraVescent Technology in six steps (Pather et al., 2003). The citric acid first dissolves in the saliva causing the $\mathrm{pH}$ to decrease to facilitate drug absorption through polar route which eliminates the use of surfactant or solvent properties to decrease the lipid characteristics of the mucosa. Once bicarbonate neutralizes the acid and effervesces, the $\mathrm{pH}$ in the oral cavity increase when carbon dioxide escapes, the protonated drug thus becomes increasingly unionized and is absorbed via nonpolar route (Pather et al., 2003). They claimed the OraVescent technology may be used for drugs with a pKa within the approximate range of 4-10 (Pather et al., 2003). The pKa of rivastigmine tartrate is reported as 8.62 (https://comptox.epa.gov/dashboard/dsstoxdb/results?utf8= \%E2\%9C\%93\&search=129101-54-8, 2017) and 8.85 (http:// www.ema.europa.eu/docs/en_GB/document_library/EPAR_ -x_Scientific_Discussion/human/000169/WC500032592.pdf, 2017), while that of donepezil $\mathrm{HCl}$ is 8.84 (https://comptox. epa.gov/dashboard/dsstoxdb/results?utf8=\%E2\%9C\%93\&search $=120011-70-3$, 2017). Both buccal tablets are very small, only $100 \mathrm{mg}$ (Table 2), thus the transmucosal permeability may occur in the oral cavity or in gastrointestinal tract. 


\section{Conclusions}

Rivastigmine L-tartrate and donepezil $\mathrm{HCl}$ were purchased from the same manufacturer which listed both as $\geq 98 \%$ purity by HPLC, but the product stability was significantly different (Fig. 4). FT-IR confirmed that water molecules are present in donepezil $\mathrm{HCl}$, which functioned as a catalyst (Bertuzzi, 2009) to induce premature effervescence during compounding and accelerated storage. The second contribution is that we found the relative humidity (in \%) recommended to compound this type of products, to be $10-20 \%$, (not more than 25\%) (Bertuzzi, 2009). Working in a laboratory facility with controlled RT and RH according to the climate zones during the research and development stage is highly suggested. Once potential formulation candidates are achieved, automation in mixing, tableting and packaging should be done in a low humidity and controlled room temperature environment, and subject the final products to stability study in controlled room temperature and relative humidity of the countries to be marketed.

\section{Acknowledgements \\ The authors express their gratitude to MCPHS University, CAPES Brazil (Brazil Coordination for the Improvement of Higher Education Personnel; Coordenação de Aperfeiçoamento de Pessoal de Nível Superior), and SACM (Saudi Arabian Cultural Mission) for making this project possible, and to American Association of Pharmaceutical Scientists for webinar presentation. \\ Corresponding author would like to express her gratitude to Jonathan Afshar, Justin Y. Shin, Hong Tiv, Sammy Tan, and Tong Yang for working together in a previous study, effervescent granule which inputs led to the current project.}

\section{Funding}

The project was financially supported by MCPHS University, Brazil Coordination for the Improvement of Higher Education Personnel (CAPES) and Saudi Arabian Cultural Mission (SACM).

Yosra Muhammad, Ph. D. in Medicinal Chemistry and Waleed Rizg, Master of Science in Pharmaceutics were sponsored by SACM, while Thais Dias Caputo, Jader M. Gomes, Denise Oliveira, Andiara Cardoso Peixoto, Bruna Sanches Pereira, Carolina Vazquez, and Thiago Medeiros Zacaron are master of pharmacy students in Brazil who were sponsored by CAPES to study 1 year at MCPHS UniversityBoston as exchange students.

\section{Availability of data and materials}

All data were summarized in the manuscript. The raw data will be kept for 5 years after publications. The study materials are available for purchased from the supply vendors listed in the manuscript. No human or animal subjects were involved in the study.

\section{Authors' contributions}

MCC, Ph.D. designed the experiment into three phases, traditional formulation, innovative formulation approach, and stability determination using FT-IR, DSC, and HPLC analysis. She also supervised students to collect data and edited the manuscript. YM, Ph.D. taught MCC, Ph.D. and students how to operate FTIR. CK, Ph.D. analyzed the Fourier Transform Infrared scans. DAW served as the committee member for a graduate student (Waleed Reed) and gave valuable critiques and advices for this project. All authors read and approved the final manuscript.

\section{Ethics approval and consent to participate}

No human or animal subjects were used in this study. Monica Chuong will take the responsibility as the corresponding author.

\section{Competing interests}

The authors declare that they have no competing interests.

\section{Publisher's Note}

Springer Nature remains neutral with regard to jurisdictional claims in published maps and institutional affiliations.

Received: 22 September 2017 Accepted: 4 January 2018

Published online: 03 February 2018

\section{References}

Allen LV Jr, Howard CA. Ansel's pharmaceutical dosage forms and drug delivery systems. Lippincott Williams and Wilkins; 2013. p. 214.

Aulton ME, Taylor KMG. Aulton's pharmaceutics. Edinburg: Elsevier; 2013. p. $845-8$.

Bertuzzi G. Effervescent granulation. In: Parkih. DM, ed. Handbook of pharmaceutical granulation technology. Informa, New York. 2009. pp. 323-337.

Bubb G. Postcompaction data analysis techniques. In: Celik M, editor. Pharmaceutical powder compaction technology. NY: Informa; 2011. p. 66-7. http://www.accessdata.fda.gov/scripts/cder/ob/docs/tempai.cfm. Accessed 25 Nov 2017.

http://www.ema.europa.eu/docs/en_GB/document_library/EPAR_-_Scientific_ Discussion/human/000169/WC500032592.pdf. Accessed 27 Nov 2017.

http://www.newsmax.com/FastFeatures/malic-acid-benefits-foods-highestconcentration-diet/2015/04/01/id/635741/. Accessed 25 Nov 2017.

http://www.tcichemicals.com/eshop/en/us/commodity/D4099/. Accessed Nov 25 2017.

http://www.tcichemicals.com/eshop/en/us/commodity/R0093/. Accessed 25 Nov 2017.

https://comptox.epa.gov/dashboard/dsstoxdb/results?utf8=\%E2\%9C\%93\&search= 120011-70-3. Accessed 27 Nov 2017.

https://comptox.epa.gov/dashboard/dsstoxdb/results?utf8=\%E2\%9C\%93\&search= 129101-54-8. Accessed 27 Nov 2017.

https://en.wikipedia.org/wiki/Maleic_acid. Accessed 27 Nov 2017.

New Jersey Department of Health and Senior Services Hazardous Substance Fact Sheet: maleic acid. http://nj.gov/health/eoh/rtkweb/documents/fs/1151.pdf. Accessed 27 Nov 2017.

Notoli Descriptions of Goods. https://natoli.com/about/. Accessed 25 Nov 2017.

Pather SI, Khankari R, Siebert J. OraVescent. In: Modified-release drug delivery technology: Marcel Dekker; 2003. p. 63-216.

Pharmaceutical Dosage Forms, General Chapters: Tablet Friability, Donepezil Hydrochloride, Donepezil Hydrochloride Tablets, Donepezil Hydrochloride Oral Disintegrated Tablets, Rivastigmine Tartrate Monographs, In: USP40-NF35. Rockville MD: Convention, Inc. 2017, pp. 537, 1543, 1749, 3859-63, 6058.

Rowe RC, Sheskey PJ, Quinn ME. Handbook of pharmaceutical excipients. London: Pharmaceutical Press; 2009. p. 410-3.

Shrewsbury RP. Applied pharmaceutics in contemporary compounding. Englewood: Morton Publishing; 2015. p. 250.

Silverstein RM, Webster FX, Kiemle DJ, Bryce DL. Spectrometric identification of organic compounds: Wiley; 2015. p. 71-125.

Vega ED, Colinas PA. Adsorption of fumaric and maleic acids onto hydroxyapatite: a thermodynamic study. J Argentine Chemical Society. 2009;97(2):195-206.

\section{Submit your manuscript to a SpringerOpen ${ }^{\circ}$ journal and benefit from:}

- Convenient online submission

- Rigorous peer review

- Open access: articles freely available online

- High visibility within the field

- Retaining the copyright to your article

Submit your next manuscript at $\gg$ springeropen.com 